\title{
Embedded Eigenvalues of Sturm Liouville Operators
}

\author{
Rafael R. del Río Castillo \\ IIMAS-UNAM, Apdo. Postal 20-726, Admón No. 20, Delegación Alvaro Obregón, \\ 01000 México D.F.
}

Received December 13, 1990; in revised form May 28, 1991

\begin{abstract}
In this work we study the behavior of embedded eigenvalues of SturmLiouville problems in the half axis under local perturbations. When the derivative of the spectral function is strictly positive, we prove that the embedded eigenvalues either disappear or remain fixed. In this case we show that local perturbations cannot add eigenvalues in the continuous spectrum. If the condition on the spectral function is removed then a local perturbation can add infinitely many eigenvalues.
\end{abstract}

\section{Introduction}

Let us consider selfadjoint realizations of differential expressions of the form

$$
(l u)(x)=-u^{\prime \prime}(x)+q(x) u(x), \quad x \in[0, \infty),
$$

where $q$ is a real valued, locally integrable function defined in $[0, \infty)$. The end point 0 is regular and we assume that the limit point case occurs at $\infty$. We are interested in studying the behavior of embedded eigenvalues when we add a function of compact support to the potential $q$.

In some way these local perturbations are very natural and the operators considered are very simple since they are second order ordinary differential operators. Nevertheless the behavior of embedded eigenvalues in this case is not yet completely understood.

The main hypothesis of this work is that the spectral function of the unperturbed operator should have a positive derivative. This prevents the occurrence of new eigenvalues and allow us to prove that the remaining eigenvalues cannot move.

Since we are using tools of the theory of ordinary differential equations our results hold only for one dimension. Under other assumptions on the potentials and for higher dimensions many interesting related results can be found in AgmonHerbst-Skibsted [1]. 
The organization of the present paper is as follows: in Sect. 1 we define an unperturbed operator $L$ and the corresponding perturbed operator $\widetilde{L}$ using the theorem of Gelfand-Levitan (see [9]) in a similar way as is done in [3], but here we allow the operator $L$ to have embedded eigenvalues. We end this section stating formally the result mentioned above.

In Sect. 2 with the help of several lemmas we give a proof of the theorem stated in Sect. 1. The main point is to generalize some results of [3] which allow us to prove that the eigenvalues of the perturbed operator are contained in a certain discrete set. Among other results this enables us to prove that the embedded dense point spectrum disappears under any local perturbation.

In Sect. 3 we prove that some local perturbations will remove embedded eigenvalues.

In Sect. 4 we give some examples of potentials which generate operators with embedded eigenvalues in the continuous spectrum and which have the property that any local perturbation cannot add embedded eigenvalues.

\section{Construction of the Unperturbed and Perturbed Operators}

Let us construct operators $L$ and $\tilde{L}$ in the following way. Let $\left\{\lambda_{n}\right\}_{n=1}^{\infty}$ be an arbitrary sequence of real numbers and let

$$
\varrho_{i}: \mathbb{R} \rightarrow \mathbb{R} \text { for } i=1,2,3
$$

be non-decreasing functions such that

a. $\varrho_{1}$ is absolutely continuous in $I_{n}$ for $n=1,2, \ldots$ and

$$
\left.\frac{d \varrho_{1}}{d \lambda}\right|_{\lambda \in I_{n}} \geqq N>0
$$

holds, where $I_{n} \subset \mathbb{R}$ are intervals, not necessarily disjoint, such that $\lambda_{n} \in I_{n}$, $n=1,2, \ldots$.

b.

$$
\varrho_{2}(\lambda):=\sum_{n=1}^{\infty} a_{n} H\left(\lambda-\lambda_{n}\right)
$$

where $a_{n}$ are positive numbers such that $\sum_{n=1}^{\infty} a_{n}<\infty$ and $H: \mathbb{R} \rightarrow \mathbb{R}$ is defined as follows:

$$
H(\mu):=\left\{\begin{array}{lll}
0 & \text { if } & \mu \leqq 0 \\
1 & \text { if } & \mu>0
\end{array} .\right.
$$

c. $\varrho_{3}$ is singular continuous in $I_{n}, n=1,2, \ldots$ or $\varrho_{3} \equiv 0$.

d. The function $\varrho:=\varrho_{1}+\varrho_{2}+\varrho_{3}$ satisfies the hypotheses of the theorem of Gelfand-Levitan (see [9]).

Definition of $\boldsymbol{L}$. By (d), we know that there exists a differential operator $L$ with spectral function $\varrho$, defined by the differential expression

$$
(l u)(x)=-u^{\prime \prime}(x)+q(x) u(x), \quad 0 \leqq x<\infty,
$$


where $q: \mathbb{R}^{+} \rightarrow \mathbb{R}$ is continuous, and the boundary condition

$$
u(0) \cos \alpha+u^{\prime}(0) \sin \alpha=0 \text {. }
$$

The limit point case occurs at $\infty$.

Remarks.

1. If we choose, for example

$$
\varrho_{1}(\lambda)=\left\{\begin{array}{lll}
0 & \text { if } & \lambda \leqq 0 \\
(2 \pi)^{-1} \sqrt{\lambda} & \text { if } & \lambda>0
\end{array}\right.
$$

and $a_{n}, n=1,2, \ldots$ small enough, then we can take $\left\{\lambda_{n}\right\}_{n=1}^{\infty}$ to be any arbitrary sequence of positive real numbers and the function

$$
\varrho:=\varrho_{1}+\varrho_{2}
$$

will satisfy the hypotheses of (d). See [6], p. 101.

2. The sequence $\left\{\lambda_{n}\right\}_{n=1}^{\infty}$ could be finite (we have only to choose $\lambda_{n}=\lambda_{n+1}=\lambda_{n+2} \ldots$ for some $n$ ).

3. The constant $N$ can depend on $I_{n}$.

Definition of $\tilde{\boldsymbol{L}}$. Let $v: \mathbb{R}^{+} \rightarrow \mathbb{R}$ be a locally integrable function with compact support $S \subset \mathbb{R}^{+}$. Let us define the selfadjoint operator $\widetilde{L}$ as the one generated by the differential expression

$$
\tilde{l u}=-u^{\prime \prime}+\{q(x)+v(x)\} u \quad x \in[0, \infty)
$$

and the boundary condition

$$
u(0) \cos \beta+u^{\prime}(0) \sin \beta=0 \quad \beta \in[0,2 \pi) .
$$

The limit point case occurs at $\infty$ since this happens for $L$.

We are interested to see how the embedded eigenvalues $\lambda_{n}, n=1,2, \ldots$ behave under the local perturbation $v$.

We shall prove the following result.

Theorem 1. Besides $\lambda_{n}, n=1,2, \ldots$ (which are eigenvalues of $L$ ) no other point in

$$
I=\bigcup_{n=1}^{\infty} I_{n}
$$

can be an eigenvalue of $\tilde{L}$. The spectrum of $\tilde{L}$ in $I \backslash\left\{\lambda_{n} \mid n=1,2, \ldots\right\}$ is purely absolutely continuous.

We shall need first some definitions and some lemmas.

\section{Proof of Theorem 1}

For $p \in \mathbb{R}$ and $\theta, \gamma \in[0, \pi)$, let us define the regular operator $L_{\theta}$ as the operator generated through the differential expression

$$
l u=-u^{\prime \prime}+q(x) u \quad 0 \leqq x \leqq p
$$


and the boundary conditions

$$
\begin{aligned}
& u(0) \cos \theta+u^{\prime}(0) \sin \theta=0, \\
& u(p) \cos \gamma+u^{\prime}(p) \sin \gamma=0 .
\end{aligned}
$$

Similarly we define the operator $\widetilde{L}_{\theta}^{\gamma}$ as the operator generated by the differential expression

$$
\tilde{\tau} u=-u^{\prime \prime}+\{q(x)+v(x)\} u \quad 0 \leqq x \leqq p
$$

and the boundary conditions

$$
\begin{aligned}
& u(0) \cos \theta+u^{\prime}(0) \sin \theta=0, \\
& u(p) \cos \gamma+u^{\prime}(p) \sin \gamma=0 .
\end{aligned}
$$

Lemma 1. Let $\gamma^{\prime}, \gamma \in[0, \pi), \gamma^{\prime} \neq \gamma$. If $\mu$ is an eigenvalue of $L_{\theta}^{\gamma}$ then $\mu$ cannot be an eigenvalue of $L_{\theta}^{\gamma^{\prime}}$.

Proof. Let $\phi(x, \mu)$ be the eigenfunction of $L_{\theta}^{\gamma}$ corresponding to the eigenvalue $\mu$. Assume that $\mu$ is also eigenvalue of $L_{\theta}^{\gamma^{\prime}}$ and let $\psi(x, \mu)$ be the corresponding eigenfunction.

Since $\phi$ and $\psi$ are solutions of

$$
l u=\mu u \quad 0 \leqq x \leqq p
$$

and satisfy the same boundary condition at zero their Wronskian is zero and they are linearly dependent. Hence it follows that they satisfy the same boundary condition at $p$ and we have a contradiction since $\gamma^{\prime} \neq \gamma$. Q.E.D.

This lemma holds also for $\widetilde{L}_{\theta}^{\gamma}, \widetilde{L}_{\theta}^{\prime}$.

Let $\delta$ be an arbitrary but fixed point of $[0, \pi)$ and choose $p \in \mathbb{R}$ such that $S \subset(0, p)$, where $S$ is the support of $v$. Let $\alpha$ and $\beta$ be as in the definition of $L$ and $\tilde{L}$.

Let $\left\{u_{1}(x, z), u_{2}(x, z)\right\}$ be a fundamental system of solutions of

$$
\left(l u_{k}\right)(x)=z u_{k}(x) \quad k=1,2 \text { and } 0 \leqq x<\infty
$$

such that

$$
\begin{aligned}
& u_{1}(0, z)=\sin \alpha \\
& u_{1}^{\prime}(0, z)=-\cos \alpha \\
& u_{2}(p, z)=\sin \delta \\
& u_{2}^{\prime}(p, z)=-\cos \delta
\end{aligned}
$$

holds.

Analogously, let $\left\{\tilde{u}_{1}(x, z), \tilde{u}_{2}(x, z)\right\}$ be a fundamental system of solutions of

which satisfies

$$
\left(\tilde{l} \tilde{u}_{k}\right)(x)=z \tilde{u}_{k}(x) \quad k=1,2 \quad 0 \leqq x<\infty
$$

$$
\begin{aligned}
& \tilde{u}_{1}(0, z)=\sin \beta \\
& \tilde{u}_{1}^{\prime}(0, z)=-\cos \beta, \\
& \tilde{u}_{2}(p, z)=\sin \delta \\
& \tilde{u}_{2}^{\prime}(p, z)=-\cos \delta .
\end{aligned}
$$


Now, for all $n \in \mathbb{N}$ let

$$
\begin{aligned}
A_{n}=\left\{\lambda \in I_{n} \mid W\left(u_{1}, u_{2}\right)(\lambda)=0\right. & \text { or } W\left(\tilde{u}_{1}, \tilde{u}_{2}\right)(\lambda)=0, \\
& \text { or } \left.W\left(\tilde{u}_{1}, u_{1}\right)(\lambda)=0\right\},
\end{aligned}
$$

where $W$ denotes the Wronskian evaluated for some $x>p$.

The points of $A_{n}$ are isolated since they are roots of analytic functions which are not identically zero. To see this, assume that $W\left(u_{1}, u_{2}\right)(\cdot) \equiv 0$ holds. Then it follows that every point is an eigenvalue of $L_{\alpha}^{\delta}$ and we have a contradiction. In the same way it can be seen that $W\left(\tilde{u}_{1}, \tilde{u}_{2}\right)(\cdot)$ is not identically zero.

If $\alpha \neq \beta$ or $v \neq 0$ then it follows that the spectrum of $L_{\alpha}^{\delta}$ is different from the spectrum of $\tilde{L}_{\beta}^{\delta}$ (see for example the end of [5]) and this implies, similarly to Lemma 2 of [3], that $W\left(\tilde{u}_{1}, u_{1}\right)(\cdot)$ is not identically zero.

The set $A_{n}$ depends on the boundary condition satisfied by $u_{2}$ and $\tilde{u}_{2}$ at the point $p$. In order to make this dependence explicit we shall write $A_{n}(\delta)$.

We shall denote by $\tilde{m}(z)$ the Weyl-Titchmarsh-Kodaira coefficient of $\tilde{L}$ with respect to $\tilde{u}_{1}, \tilde{u}_{2}$. By $\tilde{\varrho}$ we denote the spectral function of $\tilde{L}$.

Lemma 2. If $\alpha \neq \beta$ or $v \neq 0$ and $\lambda \in I_{n}$ is an eigenvalue of $\tilde{L}$, then $\lambda \in A_{n}(\delta)$ for every $\delta$ in $[0, \pi)$.

Proof. Assume $\lambda \notin A_{n}(\delta)$ for some $\delta \in[0, \pi)$. Similarly to Lemma 4 of [3] it can be proven that it is not possible to have

$$
\lim _{\varepsilon \downarrow 0}|\tilde{m}(\lambda+i \varepsilon)|=\infty
$$

(See Remark 4 at the end of the proof.)

On the other hand, since $\lambda$ is an eigenvalue of $\tilde{L}$ we have that $\varrho$ is discontinuous at $\lambda$ and therefore the symmetric derivative equals infinity, i.e.,

$$
\lim _{\varepsilon \downarrow 0} \frac{\tilde{\varrho}(\lambda+\varepsilon)-\tilde{\varrho}(\lambda-\varepsilon)}{2 \varepsilon}=D_{\lambda} \varrho=\infty .
$$

Since $\lambda \in I_{n} \backslash A_{n}(\delta)$ we have $W\left(\tilde{u}_{1}, \tilde{u}_{2}\right)(\lambda) \neq 0$ and from here it follows, as in the proof of Lemma 7 of [3], that

$$
\lim _{\varepsilon \downarrow 0}|\tilde{m}(\lambda+i \varepsilon)|=\infty
$$

Therefore we have a contradiction and $\lambda \in A_{n}(\delta)$. Q.E.D.

\section{Remarks}

4. The critical step in the proof of Lemma 4 of [3] is an estimate which depends on the inequality

$$
\frac{d \varrho_{1}}{d \lambda} \geqq N>0
$$

The particular form of the non-decreasing function $\varrho_{2}$ and of the boundary condition satisfied by $u_{2}, \tilde{u}_{2}$ at $p$ is not important in the proofs of Lemmas $1-4$ and 7 of [3]. In fact, to conclude that $\tilde{\varrho}$ is absolutely continuous, it is only necessary to have the inequality above and $\varrho$ continuous. 
5. If some bounded interval $I_{n}$ contains an infinite number of embedded eigenvalues of $L$, then any local perturbation is going to leave only a finite number of them. This follows from the fact that if $\lambda \in I_{n}$ is an eigenvalue of $\widetilde{L}$ then, from Lemma 2, we know that $\lambda \in A_{n}(\delta)$. Since the points of $A_{n}(\delta)$ are roots of analytic functions and $I_{n}$ is bounded we have that $A_{n}(\delta)$ is finite and, therefore, $\widetilde{L}$ has at most a finite number of eigenvalues in $I_{n}$. This happens in particular if we have a dense point spectrum in $I_{n}$. If the eigenvalues are not embedded, then for any local perturbation we may have an infinite number of eigenvalues. This happens for example if the spectrum of the unperturbed operator consists of isolated eigenvalues in a bounded interval which accumulate to a point of the interval. This is a consequence of the fact that the essential spectrum is preserved under local perturbations.

\section{Lemma 3.}

$$
\left\{\lambda \in I_{n} \mid W\left(\tilde{u}_{1}, u_{1}\right)(\lambda)=0\right\}=\bigcap_{\delta \in[0, \pi)} A_{n}(\delta)
$$

\section{Proof.}

"C": We need only to observe that $\tilde{u}_{1}, u_{1}$ do not depend on $\delta$.

"ว": Take $\lambda \in \bigcap_{\delta \in[0, \pi)} A_{n}(\delta)$ and assume $W\left(\tilde{u}_{1}, u_{1}\right)(\lambda) \neq 0$. Take $\delta_{1} \in[0, \pi)$. Then we must have $W\left(u_{1}, u_{2}\right)_{\delta_{1}}(\lambda)=0$ or $W\left(\tilde{u}_{1}, \tilde{u}_{2}\right)_{\delta_{2}}(\lambda)=0$.

We have written the subscripts $\delta_{1}, \delta_{2}$ to stress the fact that the Wronskians depend on the choice of $\delta_{1}, \delta_{2}$ since $u_{2}$ and $\tilde{u}_{2}$ do.

Without loss of generality, assume that $W_{\delta_{1}}\left(u_{1}, u_{2}\right)(\lambda)=0$ holds. This implies that $\lambda$ is an eigenvalue of $L_{\alpha}^{\delta_{1}}$. If we take $\delta_{2} \neq \delta_{1}, \delta_{2} \in[0, \pi)$, then using Lemma 1 it follows that $\lambda$ cannot be eigenvalue of $L_{\alpha}^{\delta_{2}}$ and therefore we have $W\left(\tilde{u}_{1}, \tilde{u}_{2}\right)_{\delta_{2}}(\lambda)=0$. This implies that $\lambda$ is an eigenvalue of $\widetilde{L}_{\alpha}^{\delta_{2}}$. If we choose $\delta_{3} \in[0, \pi)$ different from $\delta_{1}$ and $\delta_{2}$ we have, again using Lemma 1 , that $\lambda$ cannot be an eigenvalue of $\widetilde{L}_{\alpha}^{\delta_{3}}$ and $\lambda$ cannot be an eigenvalue of $L_{\alpha}^{\delta_{3}}$ either. This implies that

$$
W\left(\tilde{u}_{1}, \tilde{u}_{2}\right)_{\delta_{3}}(\lambda) \neq 0 \text { and } W\left(u_{1}, u_{2}\right)_{\delta_{3}} \neq 0 \text {. }
$$

Together with the assumption $W\left(\tilde{u}_{1}, u_{1}\right)(\lambda) \neq 0$ it follows that $\lambda \notin A_{n}\left(\delta_{3}\right)$ which is a contradiction. Q.E.D.

Proof of Theorem 1. Assume $\lambda \in I=\bigcup_{n=1}^{\infty} I_{n}$ is an eigenvalue of $\tilde{L}$. From Lemma 2 it follows that $\lambda \in \bigcap_{\delta \in[0, \pi)} A_{n}(\delta)$ for some $n \in \mathbb{N}$.

Using Lemma 3, we obtain that $W\left(\tilde{u}_{1}, u_{1}\right)(\lambda)=0$ holds. From here it follows that

$$
\tilde{u}_{1}(x, \lambda)=k u_{1}(x, \lambda) \text {, }
$$

where $k$ is a constant, for $x \geqq p$. Hence, $\lambda$ is an eigenvalue of $L$. Now, take an interval $I^{\prime} \subset I \backslash\left\{\lambda_{n} \mid n=1,2, \ldots\right\}$. The inequality

$$
\frac{d \varrho_{1}}{d \lambda} \geqq N>0
$$

holds in $I^{\prime}$. 
Since $\varrho_{2}$ is constant in $I^{\prime}$ we have that $\varrho=\varrho_{1}+\varrho_{2}+\varrho_{3}$ is continuous in $I^{\prime}$. From here it follows, as in [3] (see Remark 4), that $\tilde{\varrho}$ is absolutely continuous in $I^{\prime}$. Therefore $\widetilde{L}$ has purely absolutely continuous spectrum in $I \backslash\left\{\lambda_{n} \mid n=1,2, \ldots\right\}$. Q.E.D.

The function $W\left(\tilde{u}_{1}, u_{1}\right)(\cdot)$ is an entire function of order less than or equal to $\frac{1}{2}$ (see [7], Sect. 4, Prop. 1). From the factorization theorem of Hadamard we know that the roots $r_{n}$ of $W\left(\tilde{u}_{1}, u_{1}\right)(\cdot)$ satisfy

$$
\sum_{n=1}^{\infty}\left|r_{n}\right|^{\theta}<\infty
$$

for some integer $\theta>-\frac{3}{2}$.

Assume now, for example, that $L$ has embedded eigenvalues $\left\{\lambda_{n}\right\}_{n=1}^{\infty}$ and $\lim _{n \rightarrow \infty} \lambda_{n}=\infty$. If $\lambda_{n}, n=1,2, \ldots$ are also eigenvalues of the perturbed operator $\widetilde{L}$, then we must have $W\left(\tilde{u}_{1}, u_{1}\right)\left(\lambda_{n}\right)=0, n=1,2, \ldots$ and, therefore,

$$
\sum_{n}^{\infty}\left|\lambda_{n}\right|^{-1}<\infty
$$

must be satisfied.

If we choose for example $\lambda_{n}=O\left(n^{\alpha}\right)$ with $0<\alpha \leqq 1$ then condition (1) is not satisfied and therefore there are infinitely many $\lambda_{n}$ which cannot be eigenvalues of $\widetilde{L}$. In other words, infinitely many embedded eigenvalues disappear under any local perturbation.

Corollary. If $v \equiv 0$ and $\alpha \neq \beta$ then $\widetilde{L}$ has no embedded eigenvalues in I.

Proof. From the theorem it follows that $\lambda$ must be an eigenvalue of $L$ if $\lambda \in I$ is an eigenvalue or $\tilde{L}$. It can be seen directly or by applying Theorem 4 of [2] that it is not possible to have the same eigenvalue for two different boundary conditions. Therefore the conclusion of the corollary follows. Q.E.D.

\section{Another Result}

From Theorem 1 we know that local perturbations cannot add eigenvalues in $I$. The question then is whether embedded eigenvalues can disappear under these perturbations. The next theorem asserts that this happens for many perturbations but not for all. Assume $\varphi$ is an eigenfunction of $L$ with corresponding eigenvalue $\lambda$. Let $p \in \mathbb{R}$ be such that $S \subset(0, p)$, where $S$ is the support of the perturbation $v$. Let $\gamma \in[0, \pi)$ be such that

$$
\varphi(p) \cos \gamma+\varphi^{\prime}(p) \sin \gamma=0
$$

holds.

Theorem 2. $\lambda$ is an eigenvalue of $\tilde{L} \Leftrightarrow \lambda$ is an eigenvalue of $\widetilde{L}_{\beta}$.

\section{Remarks}

6. We need only assume that $L$ is in the limit point case at $\infty$ and that $\lambda$ is an eigenvalue of $L$. The theorem holds without any other assumption on the spectral function of $L$. 
7. The point $\lambda$ is an eigenvalue of $L_{\alpha}^{\gamma}$. Therefore the theorem asserts that $\lambda$ is an eigenvalue of $\tilde{L}$ if, and only if, the local perturbation is such that the regular operators $L_{\alpha}^{\gamma}$ and $\widetilde{L}_{\beta}^{\gamma}$ have the common eigenvalue $\lambda$. Therefore, not every local perturbation is going to make the eigenvalue $\lambda$ disappear but there are many for which this property holds.

\section{Proof of Theorem 2.}

$\Leftrightarrow$ Let $\tilde{\psi}$ be the eigenfunction of $\widetilde{L}_{\beta}$ corresponding to the eigenvalue $\lambda$. Let us define $\chi$ as the solution of

$$
(\ell \chi)(x)=\lambda \chi(x) \quad p \leqq x<\infty
$$

which satisfies

$$
\begin{aligned}
\chi(p) & =\tilde{\psi}(p), \\
\chi^{\prime}(p) & =\tilde{\psi}^{\prime}(p) .
\end{aligned}
$$

Now, let us construct $\tilde{\varphi}$ as follows:

$$
\tilde{\varphi}(x)=\left\{\begin{array}{lll}
\tilde{\psi}(x) & \text { if } & 0 \leqq x \leqq p \\
\chi(x) & \text { if } & p \leqq x<\infty
\end{array}\right.
$$

We shall prove that $\tilde{\varphi}$ is an eigenfunction of $\tilde{L}$ with corresponding eigenvalue $\lambda$.

Since $\tilde{\psi}$ and $\tilde{\psi}^{\prime}$ are absolutely continuous in $[0, p], \chi$ and $\chi^{\prime}$ are absolutely continuous in every bounded subinterval of $[p, \infty)$ and $\chi(p)=\tilde{\psi}(p), \chi^{\prime}(p)=\tilde{\psi}^{\prime}(p)$, it follows that $\tilde{\varphi}$ is continuously differentiable in $[0, \infty)$ and that $\tilde{\varphi}^{\prime}$ is absolutely continuous in every bounded subinterval of $[0, \infty)$.

From the definition of $\tilde{\varphi}$ it is obvious that $\tilde{\varphi}$ satisfies the boundary conditions at zero. So we have only to prove that $\tilde{\varphi}$ is in $L_{2}(0, \infty)$ in order to have that $\tilde{\varphi}$ is an eigenfunction of $\tilde{L}$.

From the construction of $\tilde{\varphi}$ it follows that

$$
\tilde{\varphi}(p) \cos \gamma+\tilde{\varphi}^{\prime}(p) \sin \gamma=\tilde{\psi}(p) \cos \gamma+\tilde{\psi}^{\prime}(p) \sin \gamma=0 .
$$

Now, the eigenfunction $\varphi$ which satisfies

$$
L \varphi=\lambda \varphi
$$

also satisfies the boundary condition (2). If we calculate the Wronskian of $\tilde{\varphi}$ and $\varphi$ at the point $p$ we obtain

$$
W(\tilde{\varphi}, \varphi)(p)=\tilde{\varphi}(p) \varphi^{\prime}(p)-\varphi(p) \tilde{\varphi}^{\prime}(p)=0
$$

since $\tilde{\varphi}$ and $\varphi$ satisfy the same boundary condition at $p$. Therefore, there exists $k$ such that

$$
\tilde{\varphi}(x)=k \varphi(x) \quad p \leqq x<\infty
$$

and, since $\varphi \in L_{2}(p, \infty)$, it follows that $\tilde{\varphi} \in L_{2}(p, \infty)$.

Now, from the definition of $\tilde{\varphi}$, it follows that

$$
\tilde{\ell} \tilde{\varphi}=\lambda \tilde{\varphi}
$$

and, therefore, $\lambda$ is an eigenvalue of $\tilde{L}$. 
$\Rightarrow$ Let us assume that $\tilde{\varphi}$ is the eigenfunction of $\tilde{L}$ corresponding to the eigenvalue $\lambda$. We know that there exists $\tilde{\alpha} \in[0, \pi)$ such that

$$
\cos \tilde{\alpha} \tilde{\varphi}(p)+\sin \tilde{\alpha} \tilde{\varphi}^{\prime}(p)=0
$$

holds.

If $\tilde{\alpha}=\gamma$ then it follows that $\lambda$ is an eigenvalue of $\widetilde{L}_{\beta}^{\gamma}$, since $\tilde{\varphi}$ satisfies in this case the boundary condition at $p$.

Assume that $\tilde{\alpha} \neq \gamma$. In this case we have that $\tilde{\varphi}$ and $\varphi$ are linearly independent functions in $[p, \infty)$ since, otherwise, we would have a constant $k$ such that

$$
\varphi(x)=k \tilde{\varphi}(x) \quad p \leqq x<\infty
$$

holds and this would imply that $\tilde{\varphi}$ and $\varphi$ satisfy the same boundary condition at $p$.

Since $\varphi$ and $\tilde{\varphi}$ are linearly independent and are solutions of

$$
(\ell u)(x)=\lambda u(x) \quad p \leqq x<\infty,
$$

it follows that for every solution $u$ of (3) there exist constants $c_{1}, c_{2}$ such that

$$
u(x)=c_{1} \tilde{\varphi}(x)+c_{2} \varphi(x) \quad p \leqq x<\infty
$$

holds.

Since $\tilde{\varphi}$ and $\varphi$ are in $L_{2}(0, \infty)$, we have

$$
\begin{aligned}
\int_{p}^{\infty}|u(t)|^{2} d t= & \int_{p}^{\infty}\left|c_{1} \tilde{\varphi}(t)+c_{2} \varphi(t)\right|^{2} d t \\
\leqq & 2 \int_{p}^{\infty}\left[\left|c_{1} \tilde{\varphi}(t)\right|^{2}+\left|c_{2} \varphi(t)\right|^{2}\right] d t \\
& <\infty,
\end{aligned}
$$

and therefore $u \in L_{2}(p, \infty)$ for every solution $u$ of (3).

Since $L$ is in the limit point case at $\infty$, we know that there always exists $u \notin L_{2}(0, \infty)$ [and therefore $\left.u \notin L_{2}(p, \infty)\right]$ such that

$$
L u=\lambda u
$$

holds (Weyl's alternative). Here we have a contradiction and, therefore, we have $\tilde{\alpha}=\gamma$ always. Q.E.D.

\section{Some Examples}

Example 1. Let $q_{0}(x)$ be a periodic potential in $[0, \infty)$. Let $\varphi_{0}(x, \lambda)$ denote the solution of the initial value problem

$$
\begin{gathered}
-u^{\prime \prime}+q_{0}(x) u=\lambda u, \\
u(0)=1, \quad u^{\prime}(0)=h_{0} .
\end{gathered}
$$

Choose $\lambda_{0} \in S$ where $S$ is the set of stability intervals and consider

$$
q(x):=q_{0}(x)-2 \alpha_{0} \frac{d}{d x}\left[\frac{\varphi_{0}^{2}\left(x, \lambda_{0}\right)}{1+\alpha_{0} \int_{0}^{x} \varphi_{0}^{2}\left(t, \lambda_{0}\right) d t}\right]
$$

where $\alpha_{0}>0$. 
Now, let us define the unperturbed operator $L$ as the one generated by the differential expression

$$
(\ell u)(x)=-u^{\prime \prime}+q(x) u \quad 0 \leqq x<\infty
$$

and the condition

$$
u^{\prime}(0)=\left(h_{0}-\alpha_{0}\right) u(0)
$$

The spectral function $\varrho$ of $L$ has the form (see Levitan $[8$, p. 45])

$$
\varrho(\lambda)=\varrho_{0}(\lambda)+\alpha_{0} H\left(\lambda-\lambda_{0}\right),
$$

where $\varrho_{0}$ is the spectral function of the operator $L_{0}$ generated by

$$
-u^{\prime \prime}+q_{0}(x) u
$$

and

$$
u^{\prime}(0)=h_{0} u(0) .
$$

In other words, the operator $L$ has the same spectrum as the operator $L_{0}$ except for the embedded eigenvalue $\lambda_{0}$.

It can be proved that

$$
\left.\frac{d \varrho_{0}}{d \lambda}\right|_{\lambda \in I_{n}^{\prime}} \geqq N_{n}>0,
$$

where $I_{n}$ is an arbitrary stability interval and $I_{n}^{\prime}$ is an arbitrary compact interval such that $I_{n}^{\prime} \subset I_{n}$.

Now, if $v(x)$ is a function with compact support and we consider the operator $\tilde{L}$ generated by the differential expression

$$
\tilde{\ell} u=-u^{\prime \prime}+\{q(x)+v(x)\} u
$$

and the boundary condition

$$
u(0) \cos \beta+u^{\prime}(0) \sin \beta=0
$$

then, from previous theorems, we know that the embedded eigenvalue $\lambda_{0}$ can disappear or remain fixed depending on how we choose $v$ and that $\widetilde{L}$ cannot have other embedded eigenvalues.

Example 2. Let $q_{0}(x)$ be a potential in $[0, \infty)$ which admits the following decomposition:

$$
q_{0}(x)=q_{1}(x)+q_{2}(x),
$$

where $q_{1}(\cdot)$ is of bounded variation in $[c, \infty)$ with $q_{1}(x) \rightarrow 0$ for $x \rightarrow \infty$,

$$
q_{2}(\cdot) \in L_{1}(c, \infty),
$$

where $c \in(0, \infty)$ (see $[10$, p. 233$])$.

It can be also be proved that

$$
\left.\frac{d \varrho_{0}}{d \lambda}\right|_{\lambda \in I} \geqq N>0,
$$

where $I$ is an arbitrary interval such that $I \subset(0, \infty)$ (see $[4]$ ). 
If we construct the operators $L$ and $\tilde{L}$ exactly as in the preceding example then, from previous results; we know that the embedded eigenvalue $\lambda_{0}$ can disappear or remain fixed but cannot move in $(0, \infty)$.

\section{References}

1. Agmon, S., Herbst, I., Skibsted, E.: Perturbation of embedded eigenvalues in the generalized $N$-Body problem. Comm. Math. Phys. 122, 411-438 (1989)

2. Aronszajn, N.: On a problem of Weyl in the theory of Singular Sturm-Liouville equations. Am. J. Math. 79, 597-610 (1957)

3. del Río Castillo, R.R.: Instability of the absolutely continuous spectrum of ordinary differential operators under local perturbations. J. Math. Anal. Appl. 142, (2) (1989)

4. del Río Castillo, R.R.: Dissertation, Frankfurt am Main 1985

5. del Río Castillo, R.R.: Singular spectrum of Sturm-Liouville operators under local perturbations. Am. J. Math. 113, 203-217 (1990)

6. Eastham, M.S.P., Kalf, H.: Schrödinger-type operators with continuous spectra. Boston, London, Melbourne: Pitman Press

7. Jörgens, K.: Spectral theory of second order ordinary differential operators. Lectures delivered at Aarhus Universitet, 1962/63

8. Levitan, B.M.: Inverse Sturm-Liouville problems. Utrecht, The Netherlands: VNU Science Press, 1987

9. Naimark, M.A.: Linear differential operators. Part II. New York: Ungar, 1967

10. Weidmann, J.: Spectral theory of ordinary differential operators. Lecture Notes in Mathematics, Vol. 1258. Berlin, Heidelberg, New York: Springer 1987

Communicated by B. Simon 
